\title{
Surface-Initiated Atom Transfer Radical Polymerization of Magnetite Nanoparticles with Statistical Poly(tert-butyl acrylate)-poly(poly(ethylene glycol) methyl ether methacrylate) Copolymers
}

\author{
Patcharin Kanhakeaw, ${ }^{1}$ Boonjira Rutnakornpituk, ${ }^{1,2}$ Uthai Wichai, ${ }^{1,2}$ \\ and Metha Rutnakornpituk ${ }^{1,2}$ \\ ${ }^{1}$ Department of Chemistry and Center of Excellence for Innovation in Chemistry, Faculty of Science, \\ Naresuan University, Phitsanulok 65000, Thailand \\ ${ }^{2}$ Center of Excellence in Biomaterials, Faculty of Science, Naresuan University, Phitsanulok 65000, Thailand \\ Correspondence should be addressed to Metha Rutnakornpituk; methar@nu.ac.th
}

Received 16 November 2014; Accepted 27 January 2015

Academic Editor: Peng Gao

Copyright (C) 2015 Patcharin Kanhakeaw et al. This is an open access article distributed under the Creative Commons Attribution License, which permits unrestricted use, distribution, and reproduction in any medium, provided the original work is properly cited.

\begin{abstract}
This work presented the surface modification of magnetite nanoparticle (MNP) with poly[( $t$-butyl acrylate)-stat-(poly(ethylene glycol) methyl ether methacrylate) $]$ copolymers (P[(t-BA)-stat-PEGMA]) via a surface-initiated "grafting from" atom transfer radical polymerization (ATRP). Loading molar ratio of $t$-BA to PEGMA was systematically varied $(100: 0,75: 25,50: 50$, and $25: 75$, resp.) such that the degree of hydrophilicity of the copolymers, affecting the particle dispersibility in water, can be fine-tuned. The reaction progress in each step of the synthesis was monitored via Fourier transform infrared spectroscopy (FTIR). The studies in the reaction kinetics indicated that PEGMA had higher reactivity than that of $t$-BA in the copolymerizations. Gel permeation chromatography (GPC) indicated that the molecular weights of the copolymers increased with the increase of the monomer conversion. Transmission electron microscopy (TEM) revealed that the particles were spherical with averaged size of $8.1 \mathrm{~nm}$ in diameter. Dispersibility of the particles in water was apparently improved when the copolymers were coated as compared to $\mathrm{P}(t$ $\mathrm{BA}$ ) homopolymer coating. The percentages of MNP and the copolymer in the composites were determined via thermogravimetric analysis (TGA) and their magnetic properties were investigated via vibrating sample magnetometry (VSM).
\end{abstract}

\section{Introduction}

Magnetite nanoparticle ( $\left.\mathrm{MNP}, \mathrm{Fe}_{3} \mathrm{O}_{4}\right)$ has been extensively studied over the past decade for uses in various biomedical applications including magnetic resonance imaging (MRI) contrast enhancement [1-6], targeted drug delivery [7-10], and hyperthermia [11-16]. However, because of their large surface area-to-volume ratio, these particles tend to agglomerate into large clusters and lose the specific properties associated with their initial nanometer dimensions [17]. Therefore, their surfaces must be coated with organic polymers [18-21] or inorganic materials [22-24] to obtain necessary stability for use in various applications. There are two approaches typically employed for surface modification of MNP with polymers. One is a "grafting to" method, involving the reaction of reactive functional groups in the polymer with complementary active groups on the particle surface $[25,26]$. In general, the grafting density of "grafted to" polymer chains is limited because of the steric hindrance effect. Another method is a "grafting from" approach, involving the use of active species on the surface to initiate polymerization and usually resulting in polymers with high grafting density $[17,27]$.

The recent progress in polymerization methods gives rise to the molecular design and synthesis of well-defined polymers 
by controlled/living radical polymerizations (CRPs), including nitroxide-mediated radical polymerization (NMRP) [28], reversible addition fragmentation chain transfer (RAFT) polymerizations [29], and atom transfer radical polymerization (ATRP) [30, 31]. Among these methods, ATRP has become one of the most successful methods for polymerization and block copolymerization of a wide range of monomers in a controlled manner. Therefore, ATRP was widely employed to modify MNP surface with well-controlled polymer because of the facile functionalization of MNP with ATRP initiating species (alkyl halides) for a surface-initiated controlled polymerization [32-34].

In the current work, we focused on the surface modification of MNP with poly[( $t$-butyl acrylate)-stat-(poly(ethylene glycol) methyl ether methacrylate) $]$ copolymers ( $\mathrm{P}[(t-\mathrm{BA})$ stat-PEGMA]) with varied monomer loading ratios (100:0, $75: 25,50: 50$, and $25: 75$, resp.) via ATRP using a "grafting from" technique (Figure 1). It was hypothesized that the hydrophilic PEGMA in the P[(t-BA)-stat-PEGMA $]$ copolymers allowed the particles to well disperse in aqueous media, which is a minimum requirement for biomedical applications, while the $t$-BA groups can be cleaved in acidic dispersion to obtain the carboxylate groups on the particle surface. These negatively charged carboxylate groups serve as additional electrostatic stabilization to the particles and also allow for further functionalization with various bioentities, such as peptide, protein, DNA, or drugs. The analysis of the MNPs with carboxylate-enriched surface and their use as nanosupports for coupling with bioentities are underway and will be discussed in the future report. In the present report, the initiator for ATRP was first covalently bound onto the surface of the particles through the combination of a ligand exchange reaction and condensation of triethoxysilane to obtain ATRP initiating sites on their surface. Then, the surface-initiated ATRP of $\mathrm{P}(t-\mathrm{BA})$ homopolymer and $\mathrm{P}[(t-$ BA)-stat-PEGMA] copolymer mediated by a copper complex was carried out. Kinetic studies were performed to reveal the reactivities of $t$-BA and PEGMA in the copolymerization. Gel permeation chromatography (GPC) was also conducted to investigate the change of the molecular weights of the copolymers as a function of time. The particle size and size distribution of the copolymer-MNP composites were evidenced using transmission electron microscopy (TEM). The magnetic properties of the composites were also investigated via vibrating sample magnetometry (VSM).

\section{Materials and Methods}

2.1. Materials. Unless otherwise stated, all reagents were used without further purification: iron(III) acetylacetonate $\left(\mathrm{Fe}(\mathrm{acac})_{3}\right), 99 \%$ (Acros), benzyl alcohol, 98\% (UNILAB), oleic acid (Fluka), ethyl-2-bromoisobutyrate (EBIB), 98\% (Aldrich), 2-bromoisobutyryl bromide (BIBB), 98\% (Acros), 3-aminopropyl triethoxysilane (APS), 99\% (Acros), triethylamine (TEA), 97\% (Carlo Erba), copper(I) bromide (CuBr), 99.99\% (Aldrich), and $N, N, N^{\prime}, N^{\prime \prime}, N^{\prime \prime}$-pentamethyldiethylenetriamine (PMDETA), 99+\% (Acros). Poly(ethylene glycol)methyl methacrylate (PEGMA, $\overline{M_{n}}=300 \mathrm{~g} / \mathrm{mol}$ )
(Aldrich) was used after removing inhibitors by a column filtration through basic and then neutral alumina and stored at $-4^{\circ} \mathrm{C}$ until used. Tert-butyl acrylate $(t$-BA, $99 \%$ stabilized) (Acros) was stirred overnight over $\mathrm{CaH}_{2}$ and distilled under reduced pressure prior to use. 2-Bromo-2-methyl- $N$ (3-(triethoxysilyl) propyl)propanamide (BTPAm) was prepared according to the previously reported procedure and the details of the syntheses are provided in the supporting information [35] in the Supplementary Material available online at http://dx.doi.org/10.1155/2015/121369. Toluene, 1,4dioxane, and $N, N$-dimethylformamide (DMF) (UNILAB) were used after distillation over $\mathrm{CaH}_{2}$.

2.2. Characterization. Proton nuclear magnetic resonance spectroscopy $\left({ }^{1} \mathrm{H}\right.$ NMR $)$ spectra were obtained from a Bruker AC 200-MHz spectrometer using $\mathrm{CDCl}_{3}$ as a solvent. Fourier transformed infrared spectroscopy (FTIR) experiments were performed on a Perkin-Elmer Model 1600 Series FTIR Spectrophotometer. The samples were prepared by mixing dried solid samples with $\mathrm{KBr}$. The molecular weights and molecular weight distributions were measured by GPC (Spectra System AS1000 autosampler) using tetrahydrofuran (THF) as a solvent and polystyrene standard. Magnetic properties of the particles were determined using a Standard 7403 Series at Lakeshore VSM. Samples for TEM analysis were prepared by drop casting the samples onto a carbon-coated copper grid and the images were taken using a Philips Tecnai 12 operated at $120 \mathrm{kV}$ equipped with a Gatan model $782 \mathrm{CCD}$ camera. Thermogravimetric analysis (TGA) was performed on SDTA 851 Mettler-Toledo at the temperature ranging between 25 and $600^{\circ} \mathrm{C}$ at a heating rate of $20^{\circ} \mathrm{C} / \mathrm{min}$ under oxygen atmosphere.

\subsection{Syntheses}

2.3.1. Synthesis of MNP Immobilized with ATRP Initiators (BTPAm-Immobilized MNP). BTPAm-immobilized MNP was synthesized via a three-step reaction: (1) synthesis of MNP core, (2) immobilizing the MNP with oleic acid, and (3) immobilizing BTPAm onto the oleic acid-immobilized MNP. MNP was prepared via a thermal decomposition reaction of $\mathrm{Fe}(\mathrm{acac})_{3}(5 \mathrm{~g}, 14.05 \mathrm{mmol})$ in benzyl alcohol $(90 \mathrm{~mL})$ [36]. The mixture was set at $180^{\circ} \mathrm{C}$ for $48 \mathrm{~h}$ with nitrogen flow. After the reaction, the precipitant was removed from the dispersion using an external magnet and washed with ethanol and $\mathrm{CH}_{2} \mathrm{Cl}_{2}$ repeatedly to remove benzyl alcohol and then dried under reduced pressure. To prepare oleic acid-immobilized MNP, MNP dispersion ( $0.8 \mathrm{~g}$ of dried MNP in $30 \mathrm{~mL}$ of toluene) was sonicated for $1 \mathrm{~h}$ followed by dropwise additions of oleic acid $(4 \mathrm{~mL})$ into the dispersion and sonicated for $3 \mathrm{~h}$ under nitrogen atmosphere. To immobilize BTPAm onto the MNP surface, the oleic acid-immobilized MNP dispersed in toluene was mixed with BTPAm using TEA and the reaction was carried out at room temperature for $24 \mathrm{~h}$ under nitrogen atmosphere. The dispersion was precipitated in methanol and washed with toluene to remove oleic acid and ungrafted BTPAm from the dispersion. 


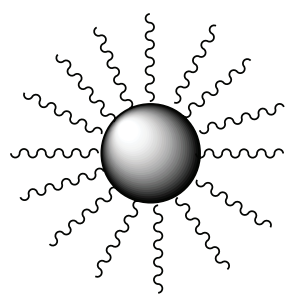

Oleic acid-coated MNP
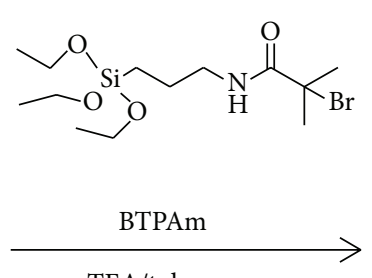

TEA/toluene

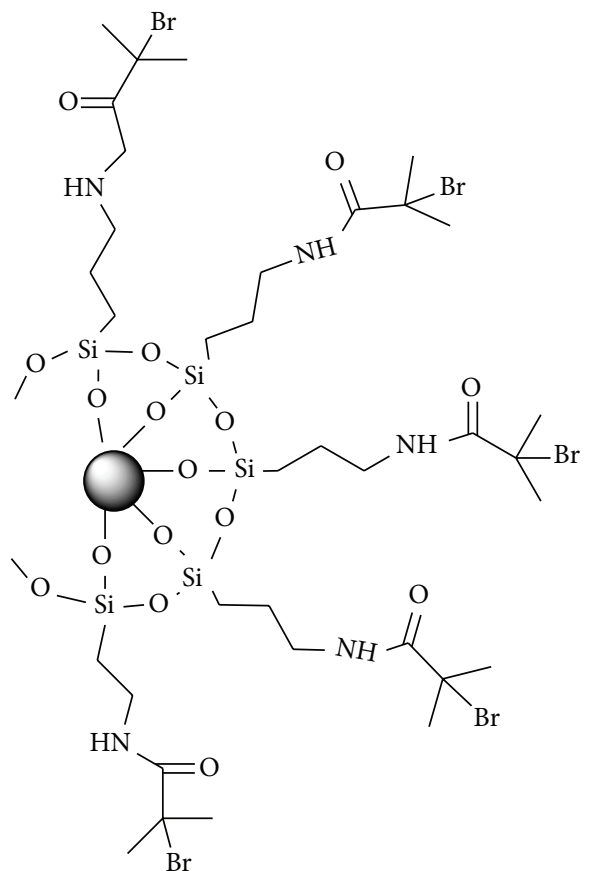

BTPAm-coated MNP

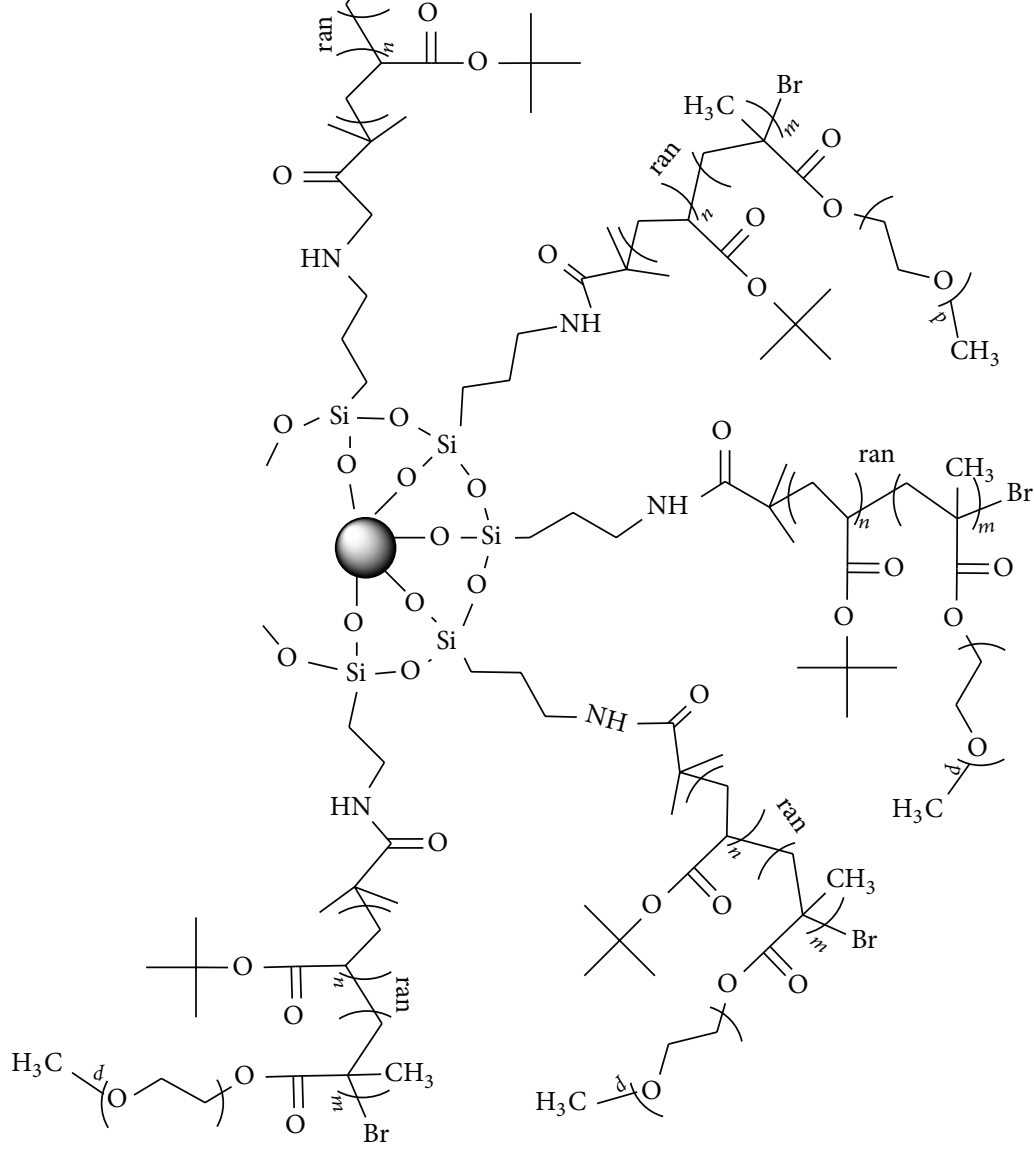

$n: m$ molar ratio in feed

$100: 0$

$75: 25$

$50: 50$

$25: 75$

$\mathrm{P}(t$-BA $) /$ PEGMA-coated MNP

FIGURE 1: Surface modification of MNP with P[( $t$-BA)-stat-PEGMA] copolymer via a surface-initiated "grafting from" ATRP reaction. 
2.3.2. Surface-Initiated ATRP of P[(t-BA)-stat-PEGMA] Copolymers on $M N P$. In the present work, MNP coated with $\mathrm{P}[(t$-BA $)$-stat-PEGMA] copolymers with various molar ratios of each composition (100:0, $75: 25,50: 50$, and $25: 75$ molar ratios of $t$-BA/PEGMA, resp.) was prepared. An example for the reaction loaded with 50:50 molar ratio of $t$-BA/PEGMA was explained. Other copolymer-MNP composites were prepared in a similar fashion with appropriate amounts of reagents used. In the typical procedure, BTPAmimmobilized MNP (0.1 g), $t$-BA $(1.45 \mathrm{~mL}, 0.01 \mathrm{~mol})$, PEGMA $(2.86 \mathrm{~mL}, 0.01 \mathrm{~mol})$, and $\mathrm{EBiB}(0.03 \mathrm{~mL}, 0.0002 \mathrm{~mol})$, used as an sacrificial initiator, were mixed in 1,4 -dioxane $(2.5 \mathrm{~mL}$, $60 \% \mathrm{w} / \mathrm{v}$ ) by ultrasonication for $15 \mathrm{~min}$. Then, the mixture was degassed by ultrasonication for $10 \mathrm{~min}$, followed by three freeze-pump-thaw cycles. PMDETA $(0.04 \mathrm{~mL}, 0.0002 \mathrm{~mol})$, $\mathrm{CuBr}(0.03 \mathrm{~g}, 0.01 \mathrm{~mol})$, and DMF $(0.22 \mathrm{~mL}, 5 \% \mathrm{v} / \mathrm{v})$, used as an internal standard, were added into the solution under nitrogen atmosphere, and it was then stirred at $90^{\circ} \mathrm{C}$ at a given time to initiate the ATRP reactions. The (co)polymer-MNP composite was removed from the dispersion by precipitation in diethyl ether, centrifugation $(35,000 \mathrm{rpm}$ for $10 \mathrm{~min})$, and magnetic separation and finally it was dried under reduced pressure.

\section{Results and Discussion}

3.1. Synthesis of MNP Immobilized with the ATRP Initiator (BTPAm-Immobilized MNP). To prepare MNP, $\mathrm{Fe}(\mathrm{acac})_{3}$ was used as iron precursor and benzyl alcohol as both reducing agent and reaction solvent. $\mathrm{Fe}^{3+}$ of $\mathrm{Fe}(\mathrm{acac})_{3}$ was partially reduced to $\mathrm{Fe}^{2+}$ by hydroxyl groups of benzyl alcohol to obtain the crystal structure of magnetite $\left(\mathrm{Fe}_{3} \mathrm{O}_{4}\right.$ or $\left.\mathrm{Fe}_{2} \mathrm{O}_{3} \cdot \mathrm{FeO}\right)$ [36]. During this process, the initial red-brown complex of $\mathrm{Fe}(\mathrm{acac})_{3}$ changed to black upon heating to $180^{\circ} \mathrm{C}$ for $48 \mathrm{~h}$, indicating the formation of MNP. After the purification process, FTIR showed a characteristic absorption peak of the $\mathrm{Fe}-\mathrm{O}$ bond at $578 \mathrm{~cm}^{-1}$ (in the supporting information). According to TEM, the particle size was in the range of 5$11 \mathrm{~nm}$ with the average of $7.78 \pm 1.58 \mathrm{~nm}$ in diameter.

Bare MNP was then immobilized with oleic acid to form well dispersible MNP in toluene. BTPAm, an ATRP initiator, was immobilized onto the oleic acid-immobilized MNP through the combination of a ligand exchange reaction and condensation of triethoxysilane to obtain the particles with ATRP initiators on their surface. Figure 2(A) exhibits FTIR characteristic absorption peaks of BTPAmimmobilized MNP: $1648 \mathrm{~cm}^{-1}$ (-NH-CO- carbonyl stretching), $1111-1019 \mathrm{~cm}^{-1}$ (Si-O stretching), $1529 \mathrm{~cm}^{-1}$ (N-H bending), and $3341 \mathrm{~cm}^{-1}$ (N-H stretching). In combination with a strong and broad signal of Fe-O bonds $\left(578 \mathrm{~cm}^{-1}\right)$, this showed that BTPAm was immobilized to the MNP surface.

\subsection{Surface-Initiated ATRP of P [(t-BA)-stat-PEGMA] Copoly-} mers on MNP. BTPAm immobilized on the particle surface was thought to be able to trigger ATRP reaction. Because the polymers grafted on the particle surface were not detectable via an NMR technique due to inherent magnetic properties of MNP, utilization of $\mathrm{EBiB}$ as a "sacrificial initiator" was employed to monitor the reaction progress. The conversion versus time plots and $\ln \left(\left[M_{0}\right] /[M]\right)$ versus time plots of ATRP of $\mathrm{P}[(t$-BA)-stat-PEGMA] copolymer were determined from the free polymers via ${ }^{1} \mathrm{H}$ NMR.

Figure 2 shows FTIR spectra of the copolymer-coated MNP having the molar ratio of $100: 0,75: 25,50: 50$, and $25: 75$ of $t$-BA/PEGMA, respectively (Figures 2(D)-2(G)). FTIR spectra of BTPAm-immobilized MNP, $t$-BA monomer, and PEGMA oligomer were also provided for comparison in Figures 2(A)-2(C). The success of the grafting reaction of the copolymers onto the particle surface was signified by the presence of the sharp and strong signals of ester groups of PEGMA and $t$-BA repeating units: $\sim 1726 \mathrm{~cm}^{-1}$ (-CO-O-) and $1147-1100 \mathrm{~cm}^{-1}$ (C-O stretching) (Figures 2(D)-2(G)). In addition, the symmetric vibration of $t$-butyl groups $(\mathrm{C}$ $\mathrm{H}$ bending $)$ in $\mathrm{P}(t-\mathrm{BA})$ as indicated by a doublet ranging between 1390 and $1365 \mathrm{~cm}^{-1}$ was clearly observed in the spectra. In all cases, a broad and strong characteristic signal of $\mathrm{Fe}-\mathrm{O}$ bonds from the MNP cores was obviously apparent $\left(\sim 587 \mathrm{~cm}^{-1}\right)$.

Kinetic studies of ATRP of $\mathrm{P}(t-\mathrm{BA})$ homopolymer $(100: 0$ of $t$-BA/PEGMA) were plotted as a function of the reaction time to show that the reaction was initially fast and its propagation rate decreased after $8 \mathrm{~h}$ of the reaction ( $480 \mathrm{~min}$ ) (Figure 3(a)). The polymerization was the first-order kinetics during the first $8 \mathrm{~h}$ of the reaction $(76 \%$ conversion after $8 \mathrm{~h}$ reaction), indicating a constant concentration of active radical species. The rate of monomer conversion started to deviate from linearity at higher monomer conversion (after $8 \mathrm{~h}$ reaction), indicating the presence of irreversible terminations.

In the cased of the copolymerizations, the percent monomer conversions of the reactions having different molar ratios of $t$-BA/PEGMA were determined from ${ }^{1} \mathrm{H} N M R$ spectra using the similar procedure to those of the $\mathrm{P}(\mathrm{t}-$ BA) homopolymer. Figures 3(b)-3(d) show the individual monomer conversion and the first-order kinetics plots of the copolymerization having various monomer molar ratios in feed. From these three copolymerizations, it can be seen that $t$-BA and PEGMA were consumed rapidly at the beginning period and slow down at the ending period. It was apparent that PEGMA was consumed more quickly than $t$-BA as indicated by the higher monomer conversion of PEGMA than that of $t$-BA at the same reaction time. For example, in the copolymerization with 50:50 molar ratio of $t$-BA/PEGMA, the conversion of PEGMA was $88 \%$, while that of $t$-BA was only $64 \%$ at $180 \mathrm{~min}$ of the reaction (Figure $3(\mathrm{c})$ ). When the reaction was prolonged, the monomer consumption deviated from linearity, indicating that the rate of the ATRP was not directly proportional to the monomer concentration. This was again probably due to the premature termination of active radicals during the copolymerization due to the decrease of the monomer concentration. This phenomenon was confirmed by the rather broad PDI for a controlled radical polymerization when the reaction time was extended (Table 1).

In addition, it was also observed that increasing PEGMA molar ratio in the dispersions seemed to promote the rate 


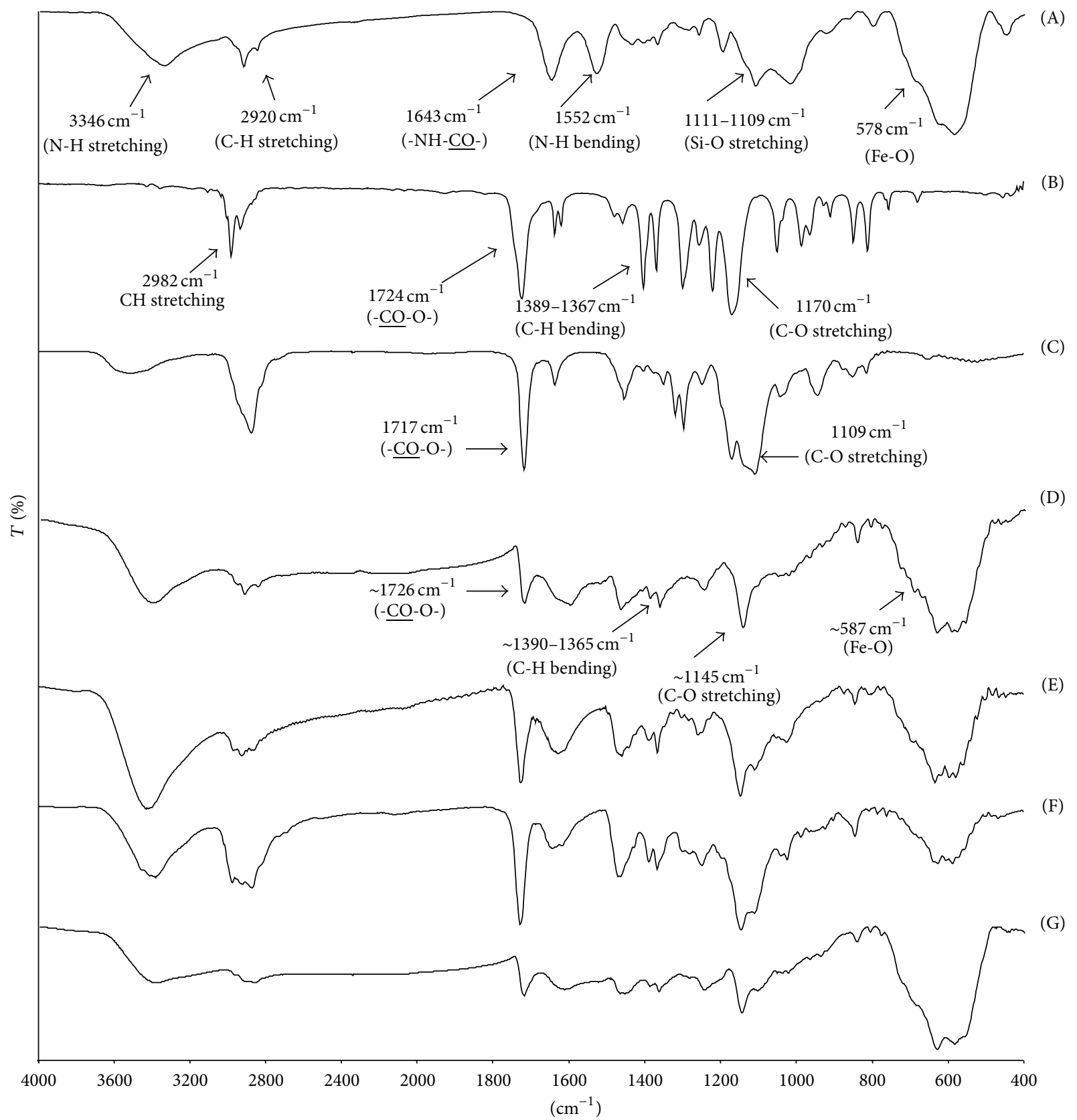

FIGURE 2: FTIR spectra of (A) BTPAm-immobilized MNP, (B) $t$-BA monomer, (C) PEGMA oligomer, and the copolymer-coated MNPs having (D) $100: 0$, (E) $75: 25$, (F) $50: 50$, and (G) $25: 75$ molar ratios of $t$-BA/PEGMA.

TABLE 1: The monomer conversion, $\bar{M}_{n}$, and PDI of P[(t-BA)-stat-PEGMA] copolymer (50/50 molar ratio) using 50/50/1/1/1 molar ratio of $[t-\mathrm{BA}]_{0} /[\mathrm{PEGMA}]_{0} /[\mathrm{EBiB}]_{0} /[\mathrm{CuBr}]_{0} /[\mathrm{PMDETA}]_{0}$ in 1,4 -dioxane at $90^{\circ} \mathrm{C}$.

\begin{tabular}{|c|c|c|c|c|c|c|}
\hline \multirow{2}{*}{ Entry } & \multirow{2}{*}{ Reaction time } & \multicolumn{2}{|c|}{$\%$ conversion $^{\mathrm{a}}$} & \multirow{2}{*}{$\bar{M}_{n}^{\mathrm{b}}(\exp )(\mathrm{g} / \mathrm{mol})$} & \multirow{2}{*}{$\bar{M}_{n}^{c}$ (theo) $(\mathrm{g} / \mathrm{mol})$} & \multirow{2}{*}{$\mathrm{PDI}^{\mathrm{b}}$} \\
\hline & & $t-\mathrm{BA}$ & PEGMA & & & \\
\hline 1 & $15 \mathrm{~min}$ & 11 & 33 & 5,898 & 5,655 & 1.3 \\
\hline 2 & $45 \mathrm{~min}$ & 24 & 55 & 13,604 & 9,788 & 1.5 \\
\hline 3 & $2 \mathrm{~h}$ & 51 & 78 & 18,300 & 14,968 & 1.6 \\
\hline 4 & $4 \mathrm{~h}$ & 72 & 93 & 21,419 & 18,564 & 1.8 \\
\hline
\end{tabular}

${ }^{a}$ It is determined via ${ }^{1} \mathrm{H}$ NMR (monomer depletion monitored relative to DMF signal, which was used as an internal standard).

${ }^{\mathrm{b}}$ It is measured via GPC (calibrated with polystyrene standard).

${ }^{c} \bar{M}_{n, \text { th }}=\left([t-\mathrm{BA}]_{0} /[\mathrm{EBiB}]_{0} \times\right.$ conv $t$ - $\left.\mathrm{BA} \times M_{t-\mathrm{BA}}\right)+\left([\mathrm{PEGMA}]_{0} /[\mathrm{EBiB}]_{0} \times\right.$ conv.PEGMA $\left.\times M_{\mathrm{PEGMA}}\right)$. 


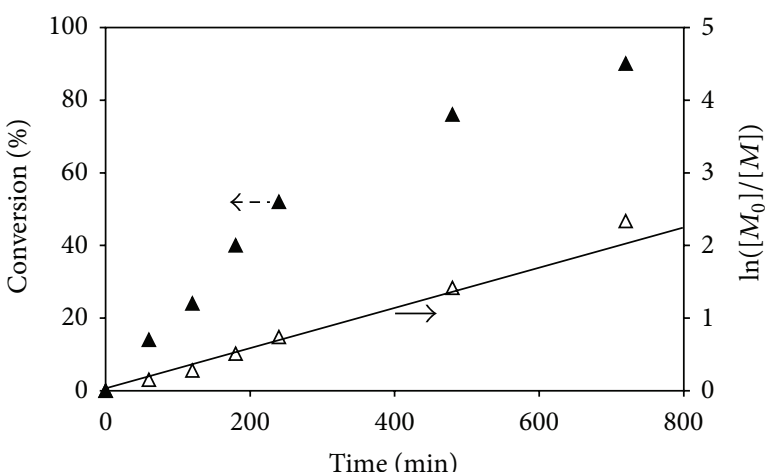

(a)

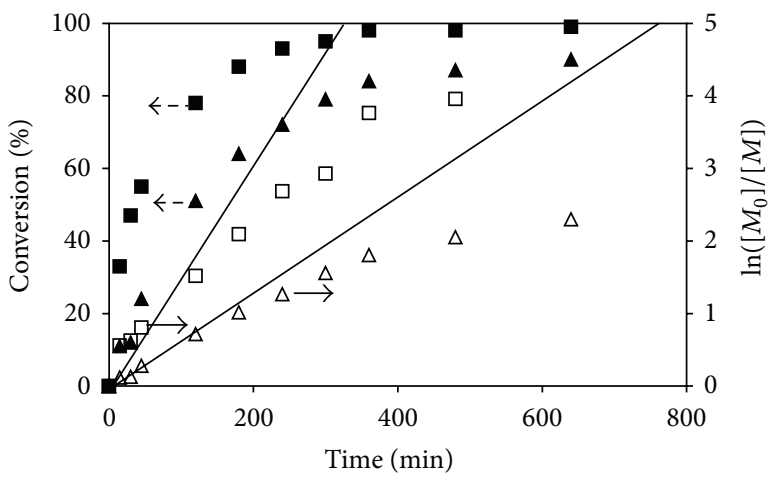

(c)

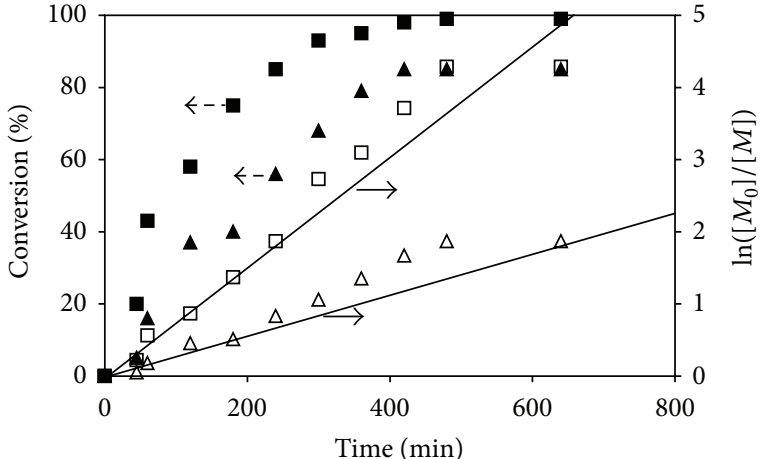

(b)

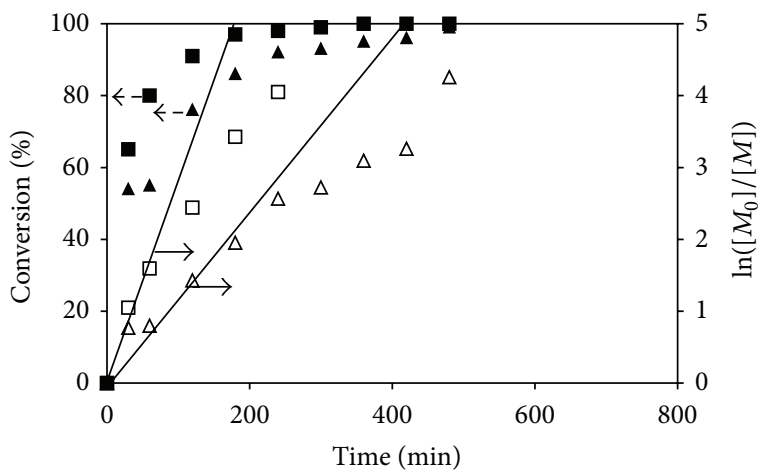

(d)

FIGURE 3: The percent conversion versus time plots and $\ln \left(\left[M_{0}\right] /[M]\right)$ versus time plots of ATRP of the copolymers having (a) 100/0, (b) $75 / 25$, (c) $50 / 50$, and (d) $25 / 75$ molar ratio of $t$-BA/PEGMA, respectively; $t$-BA $(\boldsymbol{\Lambda}, \triangle)$ and PEGMA $(\mathbf{\square}, \square)$.

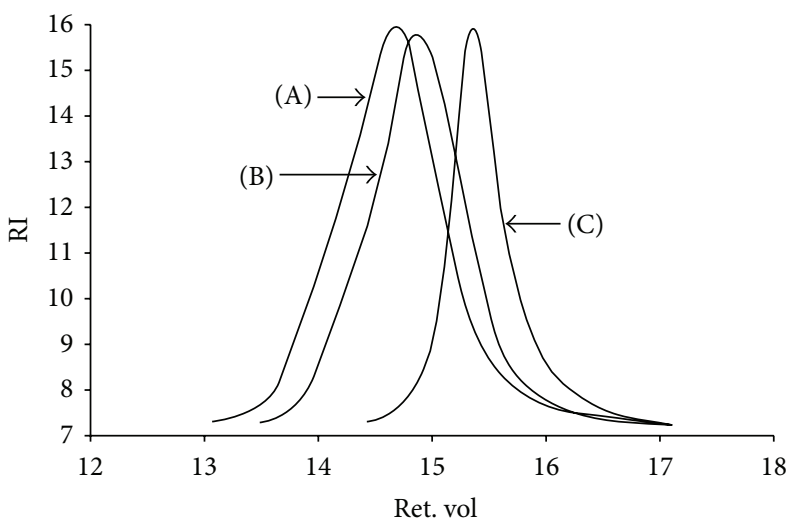
(A) $\bar{M}_{n}=18300 \mathrm{~g} / \mathrm{mol}$
(B) $\bar{M}_{n}=13604 \mathrm{~g} / \mathrm{mol}$
(C) $\bar{M}_{n}=5898 \mathrm{~g} / \mathrm{mol}$

FIGURE 4: GPC chromatograms showing molecular weight distributions of P[( $t$-BA)-stat-PEGMA] copolymers with $50: 50$ molar ratio after (A) $2 \mathrm{~h}$, (B) $45 \mathrm{~min}$, and (C) $15 \mathrm{~min}$ of the ATRP reactions.

of the ATRP reactions. For example, the copolymer with 25\% PEGMA loaded (75:25 ratio of $t$-BA/PEGMA) reached $58 \%$ PEGMA conversions after $2 \mathrm{~h}$ of the reaction. When PEGMA content in the copolymerization increased to $50 \%$ (50:50 ratio of $t$-BA/PEGMA) and 75\% (25:75 ratio of $t$ BA/PEGMA), the PEGMA conversions were $78 \%$ and $91 \%$, respectively, at the same reaction time $(2 \mathrm{~h})$. The accelerated rate of the monomer conversion was also observed in $\mathrm{P}(t$ BA) when increasing the PEGMA percentage in the copolymerization reaction. This was attributed to the increase in the solution polarity due to the increment of the PEGMA concentration in the mixture, resulting in the improvement in the copolymer solubility in 1,4-dioxane, the reaction solvent, and thus facilitating the reaction progress. 
TABLE 2: Reaction time, conversions, and the compositions of $\mathrm{P}[(t$-BA $)$-stat-PEGMA $]$ copolymers.

\begin{tabular}{|c|c|c|c|c|c|}
\hline \multirow{2}{*}{ Type of copolymer } & \multirow{2}{*}{ Reaction time (min) } & \multicolumn{2}{|c|}{$\%$ conversion $^{\mathrm{a}}$} & \multicolumn{2}{|c|}{$\%$ in the copolymer ${ }^{b}$} \\
\hline & & $t$-BA & PEGMA & $t$-BA & PEGMA \\
\hline $100: 0$ of $t$-BA/PEGMA & 240 & 52 & 0 & 100 & 0 \\
\hline $75: 25$ of $t$-BA/PEGMA & 120 & 37 & 58 & 66 & 34 \\
\hline $50: 50$ of $t$-BA/PEGMA & 120 & 42 & 64 & 40 & 60 \\
\hline $25: 75$ of $t$-BA/PEGMA & 60 & 54 & 65 & 22 & 78 \\
\hline
\end{tabular}

${ }^{\mathrm{a}}$ Reaction conversions were calculated from ${ }^{1} \mathrm{H}$ NMR spectroscopy.

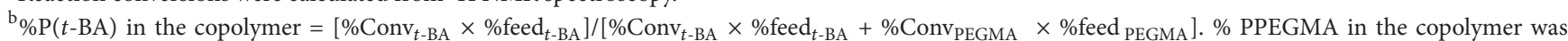
calculated in a similar fashion.
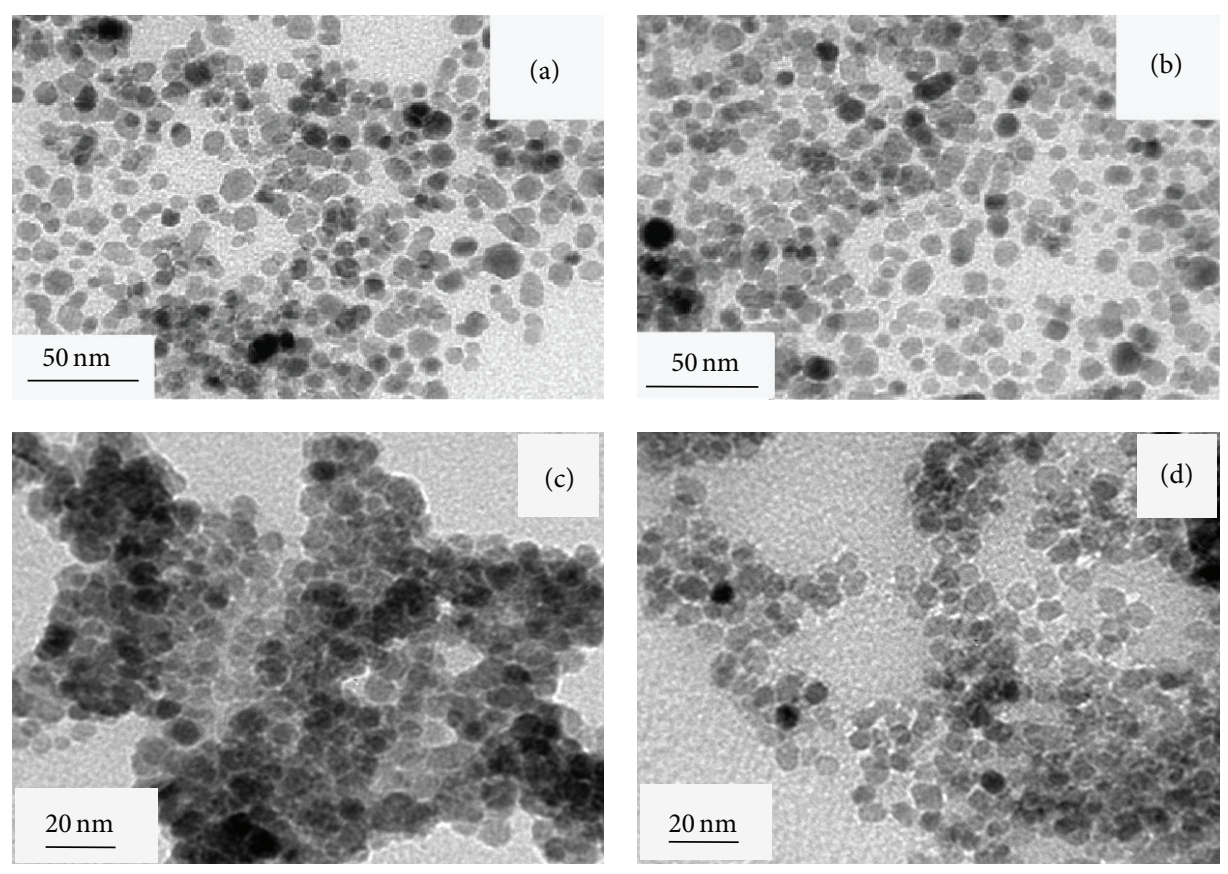

FIgURE 5: TEM images of (a) BTPAm-immobilized MNP and (b) P( $t$-BA)-coated MNP prepared from toluene dispersions and TEM images of (c) $\mathrm{P}(t-\mathrm{BA})$-coated MNP and (d) $\mathrm{P}[(t$-BA)-stat-PEGMA]-coated MNP (50:50 molar ratio) prepared from aqueous dispersions.

Table 1 reports $\bar{M}_{n}$ and PDI of P[(t-BA)-stat-PEGMA] copolymer $(50: 50$ molar ratio) at four different time intervals during the ATRP copolymerization. It was found that $\bar{M}_{n}$ gradually increased as the monomer conversions increased, indicating the growth of the copolymer chains. $\bar{M}_{n}$ calculated from ${ }^{1} \mathrm{H}$ NMR spectroscopy agreed well with those obtained from GPC, indicating the shift of $\bar{M}_{n}$ toward higher values when increasing reaction time (Figure 4). These ${ }^{1} \mathrm{HNMR}$ spectra are provided in the supporting information. The experimental $\bar{M}_{n}$ obtained from GPC experiments at different time intervals was comparable to the theoretical $\bar{M}_{n}$ calculated using the equation shown in the footnote of Table 1.

Table 2 summarizes the reaction time, percent conversion, and the estimated composition of each copolymer. The ATRP reactions were ceased at a given time corresponding to the selected percent conversion (Figure 3). These selected conversions were based on the fact that their conversions were less than $65 \%$ in order to lessen feasible reaction termination due to the depletion of the monomer concentration in the mixture. The copolymer compositions were calculated from the feed composition of the monomers, taking its percent conversion into account. Percent PEGMA in the copolymers was calculated in a similar fashion and the results were shown in Table 2. It was found that $t$-BA/PEGMA molar ratios in the copolymers were comparable to the feed compositions. Interestingly, the percentage of PEGMA in the copolymers was found to be slightly higher than its percent feeding in every composition. This result agrees well with the conversion versus time plots (Figure 3 ) indicating the higher reaction reactivity of PEGMA than that of $t$-BA. These copolymers would be then used for TEM, TGA, and VSM studies.

3.3. Properties of the MNP and the Polymer-MNP Composites. TEM images of BTPAm-immobilized MNP (from toluene), $\mathrm{P}(t-\mathrm{BA})$-coated MNP (from toluene and water), and $\mathrm{P}[(t-$ BA)-stat-PEGMA]-coated MNP (50:50 molar ratio) (from water) are shown in Figure 5. The particle size was in the range of $5-11 \mathrm{~nm}$ with the average of $8.1 \mathrm{~nm}$ in diameter, 
TABLE 3: Percentages of MNP and the copolymers in each composite.

\begin{tabular}{|c|c|c|c|c|}
\hline \multirow{2}{*}{ Type of composite } & \multirow{2}{*}{$\%$ char yield ${ }^{a}$} & \multicolumn{3}{|c|}{$\%$ in the composite } \\
\hline & & MNP & BTPAm & The copolymer \\
\hline Bare MNP & 88 & 100 & - & - \\
\hline BTPAm-immobilized MNP & 81 & 93 & 7 & - \\
\hline \multicolumn{5}{|c|}{$\mathrm{P}[(t-\mathrm{BA})$-stat-PEGMA $]$-coated MNP } \\
\hline$(100: 0)$ & 63 & 71 & 6 & 23 \\
\hline$(75: 25)$ & 55 & 63 & 5 & 32 \\
\hline$(50: 50)$ & 52 & 59 & 5 & 36 \\
\hline$(25: 75)$ & 53 & 60 & 5 & 35 \\
\hline
\end{tabular}

${ }^{\mathrm{a}}$ It is determined via TGA technique.

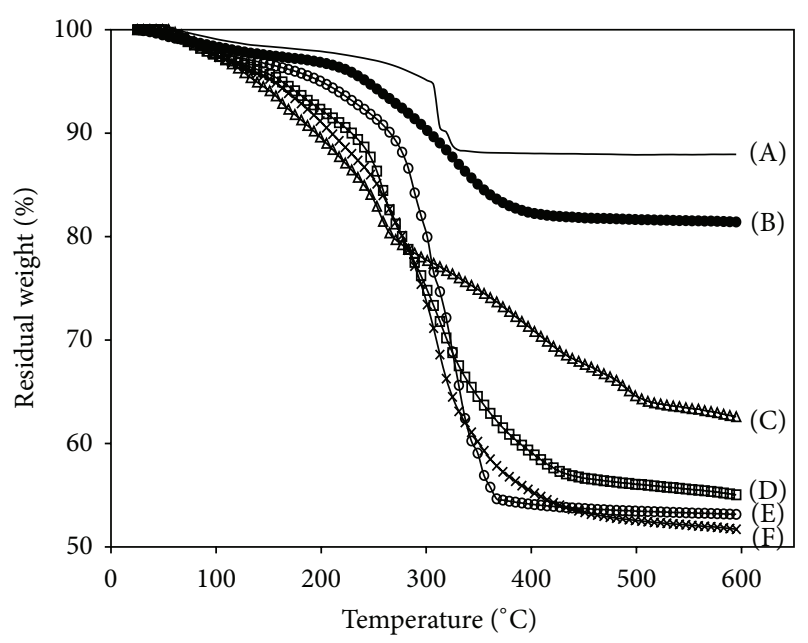

Figure 6: TGA thermograms of (A) bare MNP, (B) BTPAmimmobilized MNP, and the copolymer-coated MNP having molar ratio of $t$-BA/PEGMA of (C) $100: 0$, (D) $75: 25$, (E) $50: 50$, and (F) $25: 75$, respectively.

regardless of the dispersing media. BTPAm-immobilized MNP and $\mathrm{P}(t-\mathrm{BA})$-coated MNP were well dispersed in toluene due to the presence of hydrophobic BTPAm and $\mathrm{P}(t-$ BA) coating on the particle surface (Figures 5(a) and 5(b)). Interestingly, $\mathrm{P}(t-\mathrm{BA})$-coated $\mathrm{MNP}$ apparently aggregated when dispersed from aqueous media due to the hydrophobic $\mathrm{P}(t-\mathrm{BA})$ coating (Figure 5(c)). Dispersibility of the particles in water seemed to be much improved when $\mathrm{P}[(t-\mathrm{BA})$-statPEGMA] copolymers were used for the coating due to the increase in polarity of the coated polymer as compared to $\mathrm{P}(t$ BA) homopolymer (Figure 5(d)).

TGA studies were carried out to investigate the mass loss of the organic components in the polymer-MNP composites. The MNP in each step of the reaction showed the distinctive TGA curves, giving rise to the information of the amounts of BTPAm, the copolymers in the coated MNP (Figure 6). The slight loss in mass of bare MNP was probably due to the remaining benzyl alcohol used as the reaction solvent in the MNP preparation step. Using an assumption that $\%$ char yield was the weight of iron oxide in the form of magnetite remaining at $600^{\circ} \mathrm{C}$, the weight loss of the surfacemodified MNPs was thus attributed to the decomposition

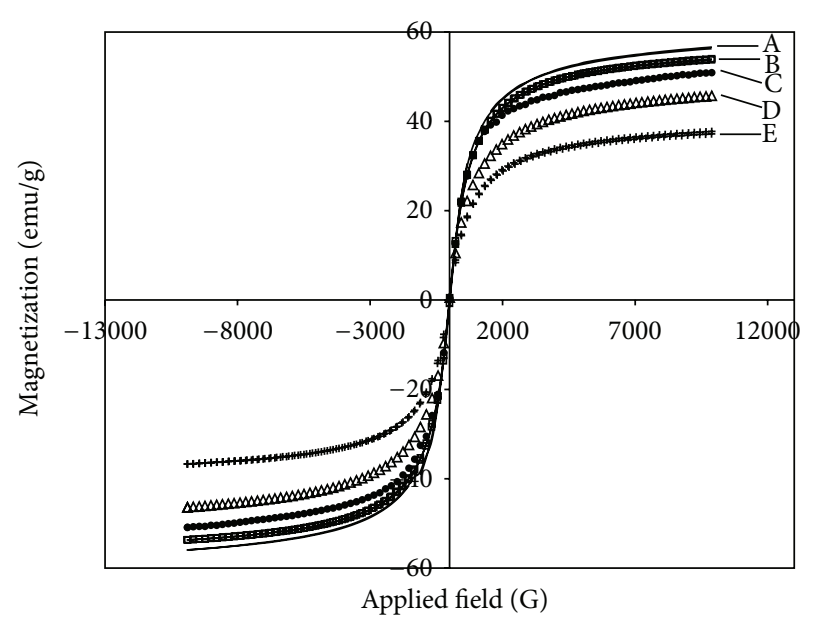

Figure 7: Magnetic properties of (A) bare MNP, (B) BTPAmimmobilized MNP, and $\mathrm{P}[(t-\mathrm{BA})$-stat-PEGMA $]$ copolymer-coated MNP (50:50 molar ratio) after (C) $15 \mathrm{~min}$, (D) $45 \mathrm{~min}$, and (E) $120 \mathrm{~min}$ of the ATRP reactions.

of organic components including BTPAm and the polymers that were coated to the particle surface. Hence, percent char yield of bare MNP and BTPAm-immobilized MNP were first determined to obtain the percentage of BTPAm in the composites, followed by the polymer-MNP composites. According to the TGA results, the percentage of BTPAm in the composites was about 5-7 wt\%, and the percentages of the copolymers in the composites ranged between $23 \%$ and $36 \%$, and those of MNPs were in the range of $59-71 \%$. The percentages of MNPs and the polymers in each composite are shown in Table 3.

Magnetic properties of the MNP composites were investigated via VSM technique. $M-H$ curves of bare MNP, BTPAmimmobilized MNP, and the MNP coated with $\mathrm{P}[(t-\mathrm{BA})$-statPEGMA] copolymer (50:50 molar ratio) were presented in Figure 7. They showed superparamagnetic behavior at room temperature as indicated by the absence of magnetic remanence $\left(M_{r}\right)$ and coercivity $\left(H_{c}\right)$ upon removing an external magnetic field. According to the results in Figure 7, the decrease of saturation magnetization $\left(M_{s}\right)$ from $56 \mathrm{emu} / \mathrm{g}$ of bare MNP to 54 of BTPAm-immobilized MNP was attributed to the existence of a thin layer of BTPAm on the 
particle surface, resulting in the decrease of the percentage of MNP core in the composite. Similarly, the $M_{s}$ values of the MNP coated with the copolymer were much lower than those of bare MNP, which was again attributed to the decrease in MNP content in the composites because of the presence of the polymeric surfactant on their surface [35]. Increasing the reaction time from $15 \mathrm{~min}$ to $120 \mathrm{~min}$ further decreased the $M_{s}$ values of the particles $(35-50 \mathrm{emu} / \mathrm{g}$ ) due to the increase in polymer percentages in the composites, resulting in the decrease in their MNP compositions and a drop of magnetic sensitivity.

\section{Conclusions}

This work presented a "grafting from" method to modify MNP surface with $\mathrm{P}[(t-\mathrm{BA})$-stat-PEGMA] copolymer via ATRP reactions. The systematic adjustment of molar ratio of $t$-BA to PEGMA in the loading was carried out in the will of fine tuning of water dispersibility of the copolymercoated MNP. The reaction reactivity of PEGMA in the ATRP reactions was higher than that of $t$-BA. Increasing PEGMA composition in the copolymerization seemed to promote the rate of the ATRP reaction under the condition used in this work $(1: 1 \mathrm{CuBr}:$ PMDETA mol: mol in 1,4-dioxane at $\left.90^{\circ} \mathrm{C}\right) . \bar{M}_{n}$ gradually increased as the monomer conversions increased, indicating the steady growth of the copolymer chains in a controllable fashion. TEM indicated that the particles were well dispersible in water due to the presence of hydrophilic PEGMA in the copolymers as compared to those coating with $\mathrm{P}(t$-BA $)$ homopolymer. There were about $23-36 \%$ of the polymers and $59-71 \%$ of MNP cores in the copolymer-MNP composites. After the surface modification, they retained the superparamagnetic properties with the decrease in magnetic responsiveness due to the presence of the copolymers.

\section{Conflict of Interests}

The authors declare that there is no conflict of interests regarding the publication of this paper.

\section{Acknowledgments}

The authors acknowledge the Thailand Research Fund (TRF) (DBG5580002) and The National Research Council of Thailand (NRCT) (R2558B050) for financial support. They also thank the Center of Excellence for Innovation in Chemistry (PERCH-CIC), Office of the Higher Education Commission, Ministry of Education, for funding and student scholarship.

\section{References}

[1] D.-B. Shieh, F.-Y. Cheng, C.-H. Su et al., "Aqueous dispersions of magnetite nanoparticles with $\mathrm{NH}_{3}^{+}$surfaces for magnetic manipulations of biomolecules and MRI contrast agents," Biomaterials, vol. 26, no. 34, pp. 7183-7191, 2005.

[2] M. Kumagai, M. R. Kano, Y. Morishita et al., "Enhanced magnetic resonance imaging of experimental pancreatic tumor in vivo by block copolymer-coated magnetite nanoparticles with TGF- $\beta$ inhibitor," Journal of Controlled Release, vol. 140, no. 3, pp. 306-311, 2009.

[3] Y. M. Wang, X. Cao, G. H. Liu et al., "Synthesis of $\mathrm{Fe}_{3} \mathrm{O}_{4}$ magnetic fluid used for magnetic resonance imaging and hyperthermia," Journal of Magnetism and Magnetic Materials, vol. 323, no. 23, pp. 2953-2959, 2011.

[4] G. Liu, R. Y. Hong, L. Guo, Y. G. Li, and H. Z. Li, "Preparation, characterization and MRI application of carboxymethyl dextran coated magnetic nanoparticles," Applied Surface Science, vol. 257, no. 15, pp. 6711-6717, 2011.

[5] Z. Liao, H. Wang, R. Lv et al., "Polymeric liposomes-coated superparamagnetic iron oxide nanoparticles as contrast agent for targeted magnetic resonance imaging of cancer cells," Langmuir, vol. 27, no. 6, pp. 3100-3105, 2011.

[6] J.-J. Yuan, S. P. Armes, Y. Takabayashi et al., "Synthesis of biocompatible poly[2-(methacryloyloxy)ethyl phosphorylcholine]-coated magnetite nanoparticles," Langmuir, vol. 22, no. 26, pp. 10989-10993, 2006.

[7] M. Rutnakornpituk, S. Meerod, B. Boontha, and U. Wichai, "Magnetic core-bilayer shell nanoparticle: a novel vehicle for entrapmentof poorly water-soluble drugs," Polymer, vol. 50, no. 15, pp. 3508-3515, 2009.

[8] S. Dandamudi and R. B. Campbell, "The drug loading, cytotoxicty and tumor vascular targeting characteristics of magnetite in magnetic drug targeting," Biomaterials, vol. 28, no. 31, pp. 46734683, 2007.

[9] M. Kamat, K. El-Boubbou, D. C. Zhu et al., "Hyaluronic acid immobilized magnetic nanoparticles for active targeting and imaging of macrophages," Bioconjugate Chemistry, vol. 21, no. 11, pp. 2128-2135, 2010.

[10] K. Cheng, S. Peng, C. Xu, and S. Sun, "Porous hollow $\mathrm{Fe}_{3} \mathrm{O}_{4}$ nanoparticles for targeted delivery and controlled release of cisplatin," Journal of the American Chemical Society, vol. 131, no. 30, pp. 10637-10644, 2009.

[11] Z. Li, M. Kawashita, N. Araki, M. Mitsumori, M. Hiraoka, and M. Doi, "Magnetite nanoparticles with high heating efficiencies for application in the hyperthermia of cancer," Materials Science and Engineering C, vol. 30, no. 7, pp. 990-996, 2010.

[12] A. A. M. Elsherbini, M. Saber, M. Aggag, A. El-Shahawy, and H. A. A. Shokier, "Magnetic nanoparticle-induced hyperthermia treatment under magnetic resonance imaging," Magnetic Resonance Imaging, vol. 29, no. 2, pp. 272-280, 2011.

[13] T. Kikuchi, R. Kasuya, S. Endo et al., "Preparation of magnetite aqueous dispersion for magnetic fluid hyperthermia," Journal of Magnetism and Magnetic Materials, vol. 323, no. 10, pp. 12161222, 2011.

[14] J. Qu, G. Liu, Y. Wang, and R. Hong, "Preparation of $\mathrm{Fe}_{3} \mathrm{O}_{4}$ chitosan nanoparticles used for hyperthermia," Advanced Powder Technology, vol. 21, no. 4, pp. 461-467, 2010.

[15] A. Ito, M. Fujioka, T. Yoshida et al., "4-S-Cysteaminylphenolloaded magnetite cationic liposomes for combination therapy of hyperthermia with chemotherapy against malignant melanoma," Cancer Science, vol. 98, no. 3, pp. 424-430, 2007.

[16] R. Hergt, S. Dutz, R. Müller, and M. Zeisberger, "Magnetic particle hyperthermia: nanoparticle magnetism and materials development for cancer therapy," Journal of Physics Condensed Matter, vol. 18, no. 38, pp. S2919-S2934, 2006.

[17] H. Dong, J. Huang, R. R. Koepsel, P. Ye, A. J. Russell, and K. Matyjaszewski, "Recyclable antibacterial magnetic nanoparticles grafted with quaternized poly(2-(dimethylamino)ethyl methacrylate) brushes," Biomacromolecules, vol. 12, no. 4, pp. 1305-1311, 2011. 
[18] S. M. Kang, I. S. Choi, K. B. Lee, and Y. Kim, "Bioconjugation of poly(poly(ethylene glycol) methacrylate)-coated iron oxide magnetic nanoparticles for magnetic capture of target proteins," Macromolecular Research, vol. 17, no. 4, pp. 259-264, 2009.

[19] M. Rudolph and U. A. Peuker, "Coagulation and stabilization of sterically functionalized magnetite nanoparticles in an organic solvent with different technical polymers," Journal of Colloid and Interface Science, vol. 357, no. 2, pp. 292-299, 2011.

[20] S. Meerod, G. Tumcharern, U. Wichai, and M. Rutnakornpituk, "Magnetite nanoparticles stabilized with polymeric bilayer of poly(ethylene glycol) methyl ether-poly( $\varepsilon$-caprolactone) copolymers," Polymer, vol. 49, no. 18, pp. 3950-3956, 2008.

[21] L. Jiang, W. Sun, and J. Kim, "Preparation and characterization of $\omega$-functionalized polystyrene-magnetite nanocomposites," Materials Chemistry and Physics, vol. 101, no. 2-3, pp. 291-296, 2007.

[22] C. Liu, J. Guo, W. Yang, J. Hu, C. Wang, and S. Fu, "Magnetic mesoporous silica microspheres with thermo-sensitive polymer shell for controlled drug release," Journal of Materials Chemistry, vol. 19, no. 27, pp. 4764-4770, 2009.

[23] J. Zhou, L. Meng, Q. Lu, J. Fu, and X. Huang, "Superparamagnetic submicro-megranates: $\mathrm{Fe}_{3} \mathrm{O}_{4}$ nanoparticles coated with highly cross-linked organic/inorganic hybrids," Chemical Communications, no. 42, pp. 6370-6372, 2009.

[24] M. Garza-Navarro, A. Torres-Castro, V. González, U. Ortiz, and E. de la Rosa, "Magnetite and magnetite/silver core/shell nanoparticles with diluted magnet-like behavior," Journal of Solid State Chemistry, vol. 183, no. 1, pp. 99-104, 2010.

[25] J. Huang, R. R. Koepsel, H. Murata et al., "Nonleaching antibacterial glass surfaces via 'grafting onto': the effect of the number of quaternary ammonium groups on biocidal activity," Langmuir, vol. 24, no. 13, pp. 6785-6795, 2008.

[26] N. Pothayee, S. Balasubramaniam, R. M. Davis et al., "Synthesis of 'ready-to-adsorb' polymeric nanoshells for magnetic iron oxide nanoparticles via atom transfer radical polymerization," Polymer, vol. 52, no. 6, pp. 1356-1366, 2011.

[27] K. Matyjaszewski, H. Dong, W. Jakubowski, J. Pietrasik, and A. Kusumo, "Grafting from surfaces for 'everyone': ARGET ATRP in the presence of air," Langmuir, vol. 23, no. 8, pp. 4528-4531, 2007.

[28] C. J. Hawker, A. W. Bosman, and E. Harth, "New polymer synthesis by nitroxide mediated living radical polymerizations," Chemical Reviews, vol. 101, no. 12, pp. 3661-3688, 2001.

[29] C. Boyer, V. Bulmus, T. P. Davis, V. Ladmiral, J. Liu, and S. Perrier, "Bioapplications of RAFT polymerization," Chemical Reviews, vol. 109, no. 11, pp. 5402-5436, 2009.

[30] K. Matyjaszewski and J. Xia, "Atom transfer radical polymerization," Chemical Reviews, vol. 101, no. 9, pp. 2921-2990, 2001.

[31] W. A. Braunecker and K. Matyjaszewski, "Controlled/living radical polymerization: features, developments, and perspectives," Progress in Polymer Science, vol. 32, no. 1, pp. 93-146, 2007.

[32] P. Theamdee, B. Rutnakornpituk, U. Wichai, and M. Rutnakornpituk, "Recyclable magnetic nanoparticle grafted with $\mathrm{pH}$-responsive polymer for adsorption with DNA," Journal of Nanoparticle Research, vol. 16, pp. 2494-2506, 2014.

[33] Y. Pray-In, B. Rutnakornpituk, U. Wichai, T. Vilaivan, and M. Rutnakornpituk, "Hydrophilic azlactone-functionalized magnetite nanoparticle for conjugation with folic acid," Journal of Nanoparticle Research, vol. 16, no. 4, article 2357, 2014.

[34] T. Theppaleak, M. Rutnakornpituk, U. Wichai, T. Vilaivan, and B. Rutnakornpituk, "Anion-exchanged nanosolid support of magnetic nanoparticle in combination with PNA probes for DNA sequence analysis," Journal of Nanoparticle Research, vol. 15, no. 12, pp. 2106-2117, 2013.

[35] M. Rutnakornpituk, N. Puangsin, P. Theamdee, B. Rutnakornpituk, and U. Wichai, "Poly(acrylic acid)-grafted magnetic nanoparticle for conjugation with folic acid," Polymer, vol. 52, no. 4, pp. 987-995, 2011.

[36] N. Pinna, S. Grancharov, P. Beato, P. Bonville, M. Antonietti, and M. Niederberger, "Magnetite nanocrystals: nonaqueous synthesis, characterization, and solubility," Chemistry of Materials, vol. 17, no. 11, pp. 3044-3049, 2005. 

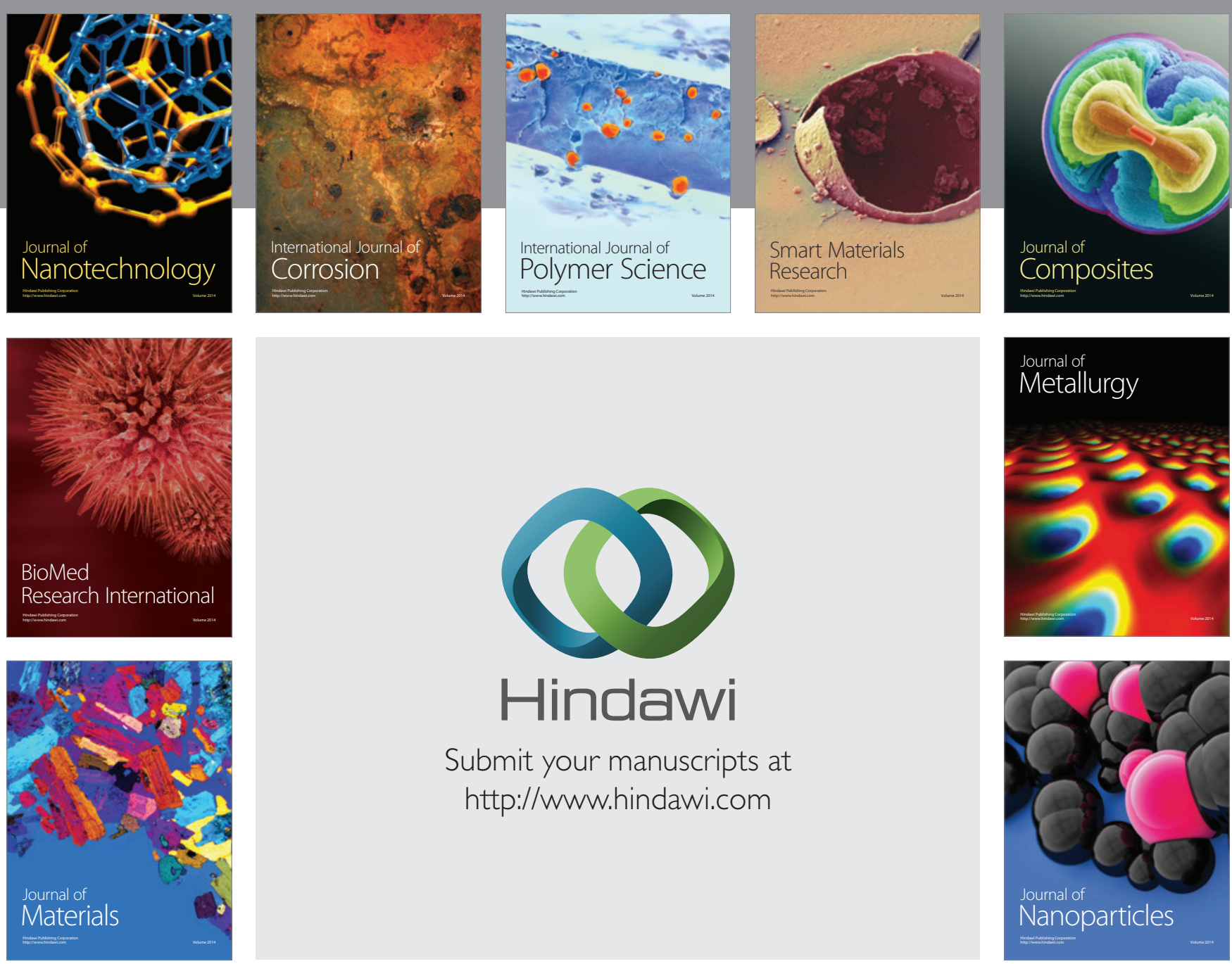

Submit your manuscripts at http://www.hindawi.com
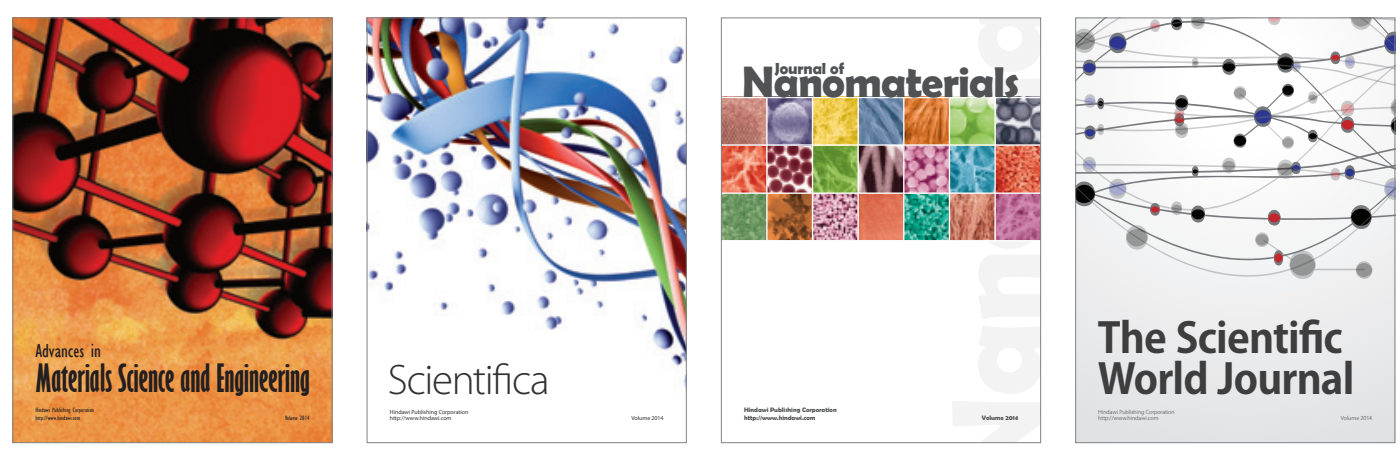

\section{The Scientific World Journal}
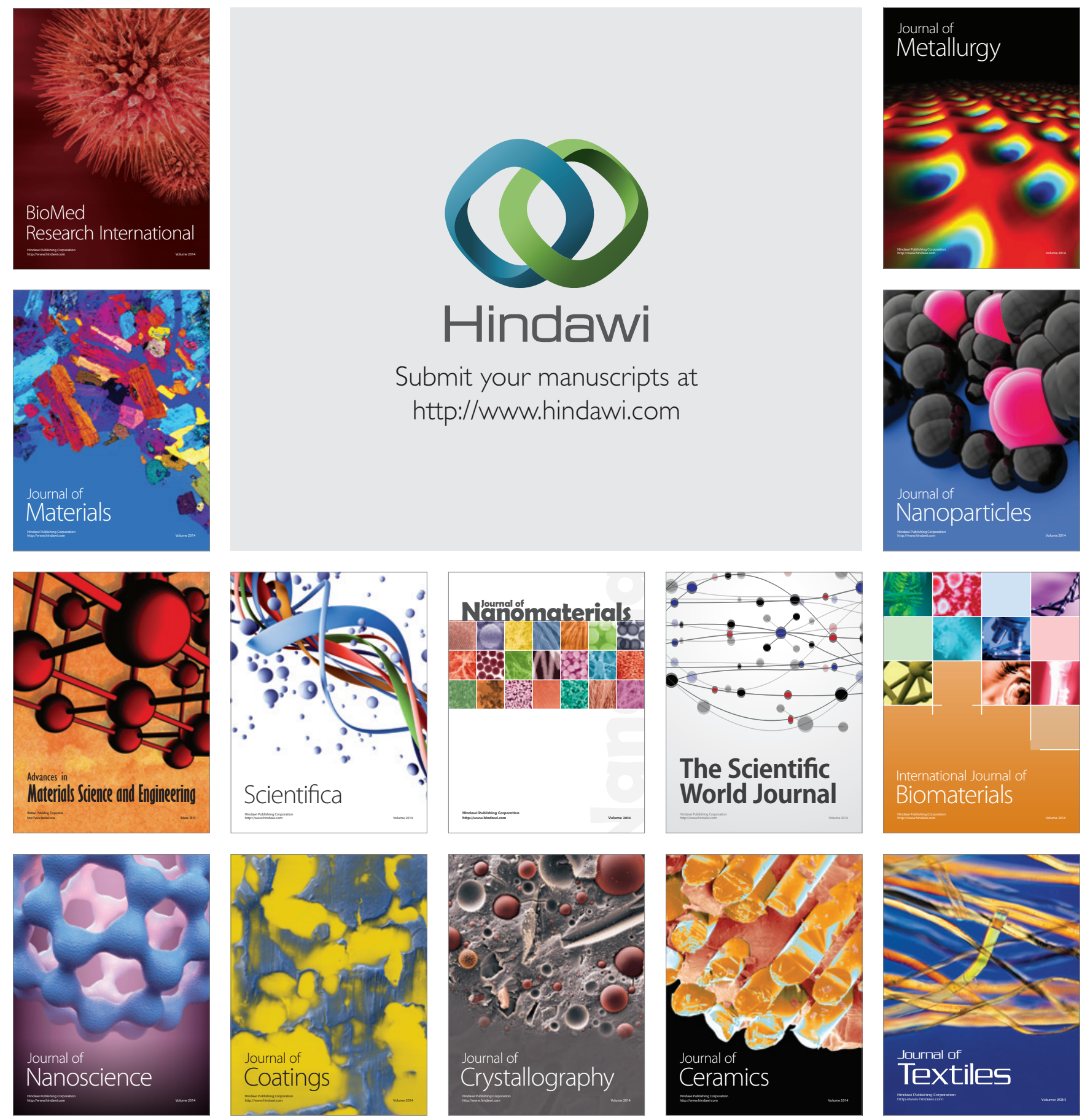